\title{
Factores socioculturales y su incidencia en la violencia contra la mujer en casos atendidos del Centro Emergencia Mujer Huancané
}

\author{
Sociocultural factors and their incidence in violence \\ against women in cases attended by the Huancane \\ women's emergency center
}

\author{
Gregoria Fernández Sanchoํㅗㄴ Martha Palomino Coila², Soledad Jackeline Zegarra Ugarte ${ }^{3}$
}

\begin{abstract}
Resumen
La violencia contra la mujer es un problema que atañe a la sociedad contemporánea en diversos ámbitos. Inciden en su manifestación diversos factores. La presente investigación tuvo como objetivo general determinar la incidencia de los Factores Socioculturales en la violencia contra la mujer en casos atendidos en el Centro Emergencia Mujer Huancané -2017. El diseño fue de tipo básico - teórico que correspondió al nivel correlacional-causal. Se enmarca dentro del enfoque cuantitativo, utiliza el método hipotético deductivo y fue no experimental de corte transversal. Para el procesamiento de datos, se utilizó el diseño estadístico Chi cuadrado, como técnica se utilizó la encuesta con su instrumento cuestionario estructurado nominal. La población estuvo constituida por 108 mujeres víctimas de violencia. Los resultados demostraron que los factores socioculturales inciden significativamente en la violencia contra la mujer con un valor de x2 $(28,664>5,9915)$. Entre estos factores se encuentran los estereotipos que subordinan a la mujer respecto al varón.
\end{abstract}

Palabras Clave: Factores culturales, factores sociales, incidencia, mujer, violencia.

\begin{abstract}
Violence against women is a problem that affects contemporary society in several areas. Various factors influence its manifestation. The general objective of this research was to determine the incidence of sociocultural factors in violence against women in cases attended at the Huancané Women's Emergency Center -2017. The design was of a basic - theoretical type that corresponded to the correlational - causal level. It was framed within the quantitative approach, used the hypothetical-deductive method and was non-experimental cross-sectional. For data processing, the Chi-square statistical design was used, and the survey with its nominal structured questionnaire instrument was used as a technique. The population consisted of 108 women victims of violence. The results showed that sociocultural factors have a significant influence on violence against women with a value of x2 (28.664 > 5.9915). Among these factors are stereotypes that subordinate women to men.
\end{abstract}

Keywords: Cultural factors, social factors, incidence, women, violence.

\section{Introducción}

La presente investigación, titulada "Factores socioculturales y su incidencia en la violencia contra la mujer en casos atendidos del Centro Emergencia Mujer Huancané-2017”, aborda los factores que inciden en la violencia contra la mujer desde la perspectiva social y cultural, explica el desarrollo de factores intervinientes en la violencia contra la mujer desde la perspectiva social y cultural.
Desde la perspectiva social, se identifica la diferencia de roles, educación sexista y consumo de bebidas alcohólicas. Desde la perspectiva cultural se identifica el machismo, los estereotipos de género e influencia de los medios de comunicación.

En ese marco, la violencia contra la mujer se califica como un problema de salud pública y fenómeno social.

\footnotetext{
${ }^{1}$ Universidad Nacional del Altiplano Puno, Perú. 0000-0002-4255-2036, e-mail: gfs.fernandez25@gmail.com.pe

${ }^{2}$ Universidad Nacional del Altiplano Puno, Perú.0000-0003-2491-3085, e-mail: mpalomino@unap.edu.pe

${ }^{3}$ Universidad Nacional del Altiplano Puno, Perú.0000-0001-8776-9936, e-mail: sjzegarra@unap.edu.pe 
La cultura en la que se ha desarrollado la mujer, desde su niñez, ha influenciado de manera directa en su comportamiento y manera de pensar en la actualidad. El hecho de vivir en una sociedad en la que aún persiste el machismo ha constituido uno de los mayores problemas de violencia contra la mujer en los últimos tiempos.

El Plan Nacional de Erradicación de la Violencia de Género (2015) expresa que la cultura está conformada por valores, prácticas, relaciones de poder y proceso sociales, políticos y económicos, que se entrelazan en la vida cotidiana de las personas y de las comunidades creando patrones culturales que no son de manera alguna un conjunto estático y cerrado de creencias y prácticas.

En este conjunto de creencias, prácticas y de patrones culturales que fluyen y cambian llamado cultura, existen actitudes o conductas que perpetúan y generan prácticas que entrañan violencia o coacción basada en género, puesto que se reproducen en la atribución de funciones estereotipadas a hombres (niños, jóvenes y adultos) y mujeres (niñas, jóvenes y adultas) en las que se considera a niños, niñas adolescentes y mujeres, como subordinados. De manera que los estereotipos y roles expuestos para cada sexo influyen de manera directa durante el crecimiento tanto de la mujer como del hombre, dependiendo del ambiente en el que se desarrolla, los valores que le han sido inculcados desde la infancia definirán su personalidad y actitud en su vida adulta. Las creencias idealizadas que se tienen del poder superior del hombre han constituido un obstáculo a nivel general para el desarrollo de la mujer y ha perpetuado la violencia dentro del hogar, constituyéndose en un problema a nivel social y de salud pública.

El Plan Nacional de Erradicación de la Violencia de Género expone que algunas de estas actitudes o creencias tienen que ver con la serie de mitos que (re) producen la creencia en la superioridad del sexo masculino que resulta en una serie de privilegios. La más extendida es la híper necesidad sexual masculina que está relacionada al desempeño sexual para determinar la mayor o menor hombría. La explotación sexual de niños, niñas y adolescentes, así como la prostitución forzada de mujeres adultas, encuentran en esta creencia una fuente de naturalización. "La violencia sexual es también más frecuente allí donde impera un firme convencimiento de los derechos sexuales del varón o los roles de los sexos son rígidos. (Presidente Constitucional de la República, 2007).

\section{Método}

La investigación realizada corresponde al enfoque cuantitativo y el tipo de investigación es básico-teórica que corresponde a la correlacional causal, el método hipotético - deductivo, el cual permite la recolección de datos para probar hipótesis y está definido como "el camino o proceso que sigue el investigador para hacer de su actividad una práctica científica; tiene diversos pasos esenciales: observaciones del fenómeno a estudiar, formando de una hipótesis para explicar dichos fenómenos para verificar o comprobar la verdad de los enunciados deducidos a partir de la experiencia” (Rodríguez et al, 2017:189).

Asimismo, el diseño corresponde al No experimental de corte transversal. Según Hernández (2014: 152), los “diseños de investigación transversal recolectan datos en un solo momento donde su propósito es describir variables y analizar su interrelación, este diseño de investigación son sin la manipulación deliberada de variables y solo recolectarán y analizarán datos en un período específico”. La muestra es no probabilística. Según los criterios, se trabajó con todo el universo que son 108 mujeres, víctimas de violencia, atendidas en el CEM. El instrumento utilizado es el cuestionario estructurado nominal.

El procesamiento de la información se realizó con la aplicación del paquete estadístico SPSS-22 y para el análisis cuantitativo de datos se trabajó a través de cuadros estadísticos que permitieron codificar y ordenar la información en relación a las variables determinadas con sus respectivos indicadores.

\section{Resultados y Discusión}

Los resultados de la investigación (los cuales se exponen a través de tablas estadísticas 
que responden a la hipótesis planteada), las interpretaciones y la discusión, expresan los datos de mayor y menor porcentaje significativo.

Tabla 1. Diferencia de roles según frecuencia de violencia contra la mujer.

\begin{tabular}{lllllllll}
\hline \multirow{2}{*}{$\begin{array}{c}\text { Frecuencia de } \\
\text { diferencia de roles }\end{array}$} & \multicolumn{4}{c}{ Frecuencia de violencia } & \multirow{2}{*}{ TOTAL } \\
\cline { 2 - 7 } & Primera vez & \multicolumn{2}{c}{ A veces } & \multicolumn{2}{c}{ Siempre } & \\
\cline { 2 - 8 } & F & $\%$ & F & $\%$ & F & $\%$ & F & $\%$ \\
\hline Nunca & 11 & $10,0 \%$ & 5 & $4,0 \%$ & 3 & $2,0 \%$ & 19 & $17,0 \%$ \\
A veces & 22 & $20,0 \%$ & 23 & $21, \%$ & 9 & $8,0 \%$ & 54 & $50,0 \%$ \\
Siempre & 12 & $11,0 \%$ & 16 & $14,0 \%$ & 7 & $6,0 \%$ & 35 & $32,0 \%$ \\
TOTAL & 45 & $41, \%$ & 44 & $40, \%$ & 19 & $17,0 \%$ & 108 & $100, \%$ \\
\hline
\end{tabular}

Fuente: Elaborado por el equipo de trabajo.

En la Tabla 01, se observa que el $21.0 \%$ manifiesta que a veces tiene diferencia de roles en su hogar y a veces son víctimas de violencia. No obstante, el 2,0\% de mujeres encuestadas manifiesta que nunca existió diferencia de roles dentro de su hogar y son víctimas de violencia siempre. En consecuencia, en relación a la diferencia de roles, según frecuencia de violencia contra la mujer, los datos coinciden con la investigación de Vargas (2017) que concluye que la violencia intrafamiliar en el distrito de Huancayo se produce a partir de la diferencia de género, que los roles se definen tanto en la familia y la sociedad tomando en cuenta el sexo con que nacen las personas. En tal sentido, el varón es propuesto al ámbito público y la mujer es postergada al ámbito doméstico. Asimismo, García (2013) corrobora esta información. Menciona que los roles de género se adquieren durante la etapa de la niñez, se realzan durante la adolescencia y se refuerzan al inicio de la vida sexual. Los autores indican que la diferencia de roles incide directamente en la violencia contra la mujer: esto porque muchos de los miembros de la familia han sido criados bajo estigmas asociados al comportamiento, condición o rasgos que tiene un individuo y genera su inclusión en un grupo social cuyos miembros son vistos como inferiores o inaceptables, los roles de género expresan la jerarquía de poder entre hombre y mujer. Se pudo demostrar que, en la población de estudio, la diferencia de roles de género incide en actitudes violentas porque estas diferencias de género sitúan a la mujer en un lugar inferior a diferencia del hombre, por lo tanto, ven las tareas del hogar clasificado en diferentes sexos, donde los roles expresan el comportamiento de los integrantes de cada hogar. En síntesis, las mujeres que son víctimas de violencia evidencian que sus actividades están ligadas a roles de género forjados por la sociedad y, si ellas no cumplen los roles asignados desde la perspectiva del varón, son víctimas de violencia.

Tabla 2. Educación sexista según frecuencia de violencia contra la mujer

\begin{tabular}{lllllllll}
\hline \multirow{2}{*}{ Educación sexista } & \multicolumn{9}{c}{ Frecuencia de violencia } & \multirow{2}{*}{ TOTAL } \\
\cline { 2 - 7 } & \multicolumn{1}{c}{ Primera vez } & \multicolumn{2}{c}{ A veces } & \multicolumn{2}{c}{ Siempre } & & \\
\cline { 2 - 7 } & $\mathrm{F}$ & $\mathrm{F}$ & $\%$ & $\mathrm{~F}$ & $\%$ & $\mathrm{~F}$ & $\%$ \\
\hline Hostil & 12 & $11,0 \%$ & 15 & $13,0 \%$ & 4 & $3,0 \%$ & 31 & $28,0 \%$ \\
Benevolente & 29 & $26,0 \%$ & 17 & $15,0 \%$ & 10 & $9,0 \%$ & 56 & $51,0 \%$ \\
Ambivalente & 4 & $3,0 \%$ & 12 & $11,0 \%$ & 5 & $4,0 \%$ & 21 & $19,0 \%$ \\
TOTAL & 45 & $41,0 \%$ & 44 & $40,0 \%$ & 19 & $17,0 \%$ & 108 & $100,0 \%$ \\
\hline
\end{tabular}

Fuente: Elaborado por el equipo de trabajo.

En la Tabla $\mathrm{N}^{\circ}$ 2, observamos que la dimensión educación sexista es caracterizada por un tratamiento desigual. Conforme al objetivo específico $\mathrm{N}^{\circ} 01$ que es establecer la incidencia de los factores sociales en la violencia contra la mujer en los casos atendidos del Centro Emergencia Mujer Huancané-2017, el 26,0 \% de la población indica que fueron criadas bajo una 
educación sexista benevolente y por primera vez son víctimas de violencia; por otro lado, el 3,0 \% de la población indican que fueron criadas bajo la educación sexista hostil y fueron víctimas de violencia siempre.

De acuerdo a los resultados adquiridos, se sostiene que la educación sexista es un factor incidente en la violencia hacia la mujer. Dichos resultados coinciden con la investigación de Vargas (2017) quien concluye que, en el distrito de Huancayo, se educa a los hijos e hijas diferenciadamente, por lo que la educación sexista se considera uno de los factores principales para que la violencia intrafamiliar se suscite.
El tipo de educación sexista benevolente es un tipo de prejuicio hacia la mujer con connotación positiva y aparentemente benefactor donde el hombre expresa el deseo de cuidar a las mujeres, pero así mismo la encasilla a cumplir ciertos roles (Navas \& Guerrero, 2016). Se observa que el sexismo benevolente incide directamente en la conducta agresiva ostentada por los agresores, por lo tanto, se considera que la educación sexista es un factor de riesgo para la perpetración de violencia contra la mujer, donde se le concibe como un ser frágil, que no tiene poder a su vez prescinde del cuidado del hombre.

Tabla 3. Machismo según frecuencia de violencia contra la mujer.

\begin{tabular}{|c|c|c|c|c|c|c|c|c|}
\hline \multirow{3}{*}{ Machismo establecido } & \multicolumn{6}{|c|}{ Frecuencia de violencia } & \multirow{2}{*}{\multicolumn{2}{|c|}{ TOTAL }} \\
\hline & \multicolumn{2}{|c|}{ Primera vez } & \multicolumn{2}{|c|}{ A veces } & \multicolumn{2}{|c|}{ Siempre } & & \\
\hline & $\mathrm{F}$ & $\%$ & $\mathrm{~F}$ & $\%$ & $\mathrm{~F}$ & $\%$ & $\mathrm{~F}$ & $\%$ \\
\hline Muy frecuente & 7 & $6,0 \%$ & 5 & $4,0 \%$ & 3 & $2,0 \%$ & 15 & $13,0 \%$ \\
\hline Frecuente & 17 & $15,0 \%$ & 27 & $25,0 \%$ & 8 & $7,0 \%$ & 52 & $48,0 \%$ \\
\hline Poco frecuente & 21 & $19,0 \%$ & 12 & $11,0 \%$ & 8 & $7,0 \%$ & 41 & $37,0 \%$ \\
\hline TOTAL & 45 & $41,0 \%$ & 44 & $40,0 \%$ & 19 & $17,0 \%$ & 108 & $100,0 \%$ \\
\hline
\end{tabular}

Fuente: elaborado por el equipo de trabajo.

En la tabla 3, en lo que respecta al objetivo específico $\mathrm{N}^{\circ} 2$, considerando el machismo según la frecuencia de violencia contra la mujer, se puede apreciar que el 25\% de la población indica que son maltratadas con frecuencia por ideas machistas del cónyuge. Así mismo, el 2.0 \% indica que son maltratadas de modo muy frecuente dentro de sus hogares porque se conservan las ideas machistas.

Los resultados coinciden con los obtenidos por Villanueva (2015). Este autor concluye que el $64 \%$ de las mujeres víctimas de violencia en el centro de salud del distrito de Caracoto sufren de violencia física y psicológica a causa de factores culturales como el machismo que se construye desde la niñez con actitudes como las niñas se educan para la obediencia, sumisión a la autoridad masculina y responsabilidad para preservar la unidad familiar; en cambio los niños crecen con la noción de desarrollo en capacidad de poder y autoridad.

En consecuencia, estos resultados revelan que las ideas machistas dentro de los hogares inciden en la violencia contra las mujeres, ya que el machismo desarrolla conductas discriminatorias en contra de las mujeres desde casa, diferenciando el modo vestir o el tipo de juguetes, enseñando a las niñas los quehaceres del hogar y los niños no privándolos de los mismos. Un ejemplo de esta estructura de violencia palpables en la cultura y el sistema patriarcal es la manera como se inculpa a la víctima, inquiriendo qué hizo mal, acaso no cocinó bien, no atendió a los niños, qué bebió, realizó un gesto o actuó mal la mujer, hechos que pudieran justificar la violencia de los hombres contra las mujeres 
Tabla 4. Estereotipos de género según frecuencia de violencia contra la mujer.

\begin{tabular}{lllllllll}
\hline \multirow{2}{*}{$\begin{array}{c}\text { Conservación de } \\
\text { creencias } \\
\text { estereotipadas }\end{array}$} & \multicolumn{9}{c}{ Frecuencia de violencia } & \multirow{2}{*}{ TOTAL } \\
\cline { 2 - 8 } & Primera vez & A veces & Siempre & & \\
\cline { 2 - 8 } & $\mathrm{F}$ & $\%$ & $\mathrm{~F}$ & $\%$ & $\mathrm{~F}$ & $\%$ & $\mathrm{~F}$ & $\%$ \\
\hline Nunca & 12 & $11,0 \%$ & 2 & $1,0 \%$ & 1 & $0,0 \%$ & 15 & $13,0 \%$ \\
A veces & 19 & $17,0 \%$ & 30 & $27,0 \%$ & 10 & $9,0 \%$ & 59 & $54,0 \%$ \\
Siempre & 14 & $12,0 \%$ & 12 & $11,0 \%$ & 8 & $7,0 \%$ & 34 & $31,0 \%$ \\
TOTAL & 45 & $41,0 \%$ & 44 & $40,0 \%$ & 19 & $17,0 \%$ & 108 & $100,0 \%$ \\
\hline
\end{tabular}

Fuente: Elaborado por el equipo de trabajo.

En la tabla $\mathrm{N}^{\circ} 04$, se observan los resultados de cómo los estereotipos de género inciden en la violencia contra la mujer. El $27.0 \%$ de población revela que a veces son agredidas por ideas de estereotipos de género en su familia, el $0.0 \%$ de mujeres encuestadas que siempre sufren de violencia indican que los motivos de violencia nunca fueron a causa de la conservación de creencias estereotipadas. Estos resultados coinciden con los obtenidos por Condori (2015), quien demuestra que el $74 \%$ de mujeres encuestadas del CEM Carabaya indica que la violencia es inherente a los estereotipos de género, donde las formas de crianza sociocultural de las familias influyen en la violencia contra la mujer en un $49 \%$.

Quesada y López (2010) señalan que las creencias estereotipadas que se reproducen y se transmiten desde que nacemos mediante los diversos procesos de socialización. Estos estereotipos son adquiridos en la cultura en la que estamos insertos e influyen en el comportamiento cotidiano de los miembros de la sociedad según el sexo al que se pertenece. Las formas de crianza estereotipadas que influyen en la violencia contra la mujer son consideradas como patrones culturales que regulan las relaciones al interior de la familia y justifican las prácticas violentas.

En base a los datos obtenidos, se constata que las creencias estereotipadas de género sustentan las conductas violentas contra la mujer, ya que los hogares de las mujeres que fueron víctimas de violencia se caracterizan porque atribuyen comportamientos y patrones a cada uno de los géneros, los cuales son transmitidos de generación en generación. Los estereotipos de género son utilizados de manera cotidiana, los cuales son reforzados con las actitudes y comportamientos diarios. Un ejemplo claro de estereotipos de género dentro de los hogares de las víctimas son las frases como "macho, macho", "los hombres no lloran", "las mujeres solo se dedican a cuidar a los hijos", "las mujeres son débiles y sumisas", "el hombre hace respetar la casa”.

Tabla 5. Medios de comunicación según frecuencia de violencia

\begin{tabular}{|c|c|c|c|c|c|c|c|c|}
\hline \multirow{3}{*}{$\begin{array}{l}\text { Influencia de los } \\
\text { medios de } \\
\text { comunicación }\end{array}$} & \multicolumn{6}{|c|}{ Frecuencia de violencia } & \multirow{2}{*}{\multicolumn{2}{|c|}{ TOTAL }} \\
\hline & \multicolumn{3}{|c|}{ Primera vez } & \multirow{2}{*}{$\begin{array}{l}\text { A veces } \\
\%\end{array}$} & \multicolumn{2}{|c|}{ Siempre } & & \\
\hline & $\mathrm{F}$ & $\%$ & $\mathrm{~F}$ & & $\mathrm{~F}$ & $\%$ & $\mathrm{~F}$ & $\%$ \\
\hline Televisión & 17 & $15,0 \%$ & 21 & $19,0 \%$ & 10 & $9,0 \%$ & 48 & $44,0 \%$ \\
\hline Emisoras radiales & 2 & $1,0 \%$ & 3 & $2,0 \%$ & 0 & $0,0 \%$ & 5 & $4,0 \%$ \\
\hline Internet & 12 & $11,0 \%$ & 13 & $12,0 \%$ & 5 & $4,0 \%$ & 30 & $27,0 \%$ \\
\hline Celular & 14 & $12,0 \%$ & 7 & $6,0 \%$ & 4 & $3,0 \%$ & 25 & $23,0 \%$ \\
\hline TOTAL & 45 & $41,0 \%$ & 44 & $40,0 \%$ & 19 & $17,0 \%$ & 108 & $100,0 \%$ \\
\hline
\end{tabular}

Fuente: Elaborado por el equipo de trabajo.

En la tabla 5, en referencia al indicador "influencia de los medios de comunicación en la frecuencia de violencia", se puede apreciar que el $19 \%$ de la población encuestada manifiesta que la televisión, como medio de comunicación, es uno de los principales agentes de violencia dentro de los hogares, cuya frecuencia de violencia es a veces. Mientras que el $0.0 \%$ de la 
población indica que las emisoras radiales son los medios de comunicación que influyen en la violencia en sus hogares, entre las cuales fueron víctimas de violencia por primera vez.

Los medios de comunicación como televisión, radio o publicidad, cumplen la función de agentes de socialización y transmiten imaginarios colectivos que legitiman la posición inferior de las mujeres y lo femenino en la sociedad; es decir, reproducen la violencia simbólica (Menéndez-Menéndez, 2014; Araneda, 2014).

Conforme a los resultados obtenidos, Vargas (2017) señala que, en la población del distrito de Huancayo, según los testimonios que obtuvieron, el papel de los medios de comunicación, principalmente la TV, es un factor influyente para que ocurra la violencia intrafamiliar, ya que distingue y define los roles que debe cumplir cada sexo. En consecuencia, los resultados del presente cuadro demuestran que los medios de comunicación son agentes poderosos que influyen en la violencia contra la mujer.

Entonces los resultados revelan que los medios de comunicación son una herramienta poderosa para expresar mensajes, reproducir prácticas y hábitos, transmitiendo ideas que justifican acciones violentas contra la mujer. En este sentido, dispersan una fuerte influencia informativa, según los datos obtenidos; la televisión y su incidencia es alarmante debido a la agresividad y violencia que muestran sus contenidos en numerosos programas que se emiten, incluso en horarios no apropiados para menores de edad. Actualmente es muy frecuente que en los programas de TV se produzcan estereotipos y roles sexistas que contribuyen a perpetuar situaciones de desigualdad, denigración hacia la mujer, de prevalencia de un sexo sobre otro y favorecen la aparición de manifestaciones de violencia.

\section{Conclusiones}

Los factores socioculturales inciden significativamente en la violencia contra la mujer en casos atendidos del Centro Emergencia Mujer Huancané -2017, bajo un valor de xc $2(28,664>5,9915)$ con 8 grados de libertad, significancia de vp (0.000), probabilidad menor a $(\alpha=0.05)$ y con un nivel de confianza del $95 \%$ que demuestra que los factores socioculturales inciden significativamente en la violencia contra la mujer, con mayor incidencia en la educación sexista y estereotipos de género.

Los factores sociales inciden significativamente en la violencia contra la mujer en casos atendidos del Centro Emergencia Mujer Huancané 2019, hallándose la educación sexista como resultado predominante de $26,0 \%$, según la prueba estadística de Chi cuadrado, entre las dos variables que muestran una probabilidad de error del 5\%, con un valor de xc $2(18,000)>$ xt 2 $(9.4877=4 \mathrm{gl})$, con una significación de vp (0.000) y probabilidad menor a $(\alpha=0.05)$ que confirman que los factores sociales como educación sexista inciden significativamente en la violencia contra la mujer, lo que se evidencia en la tabla $\mathrm{N}^{\circ} 02$ donde el 26,0 \% de mujeres encuestadas indican ser víctimas de violencia a causa de la educación sexista dentro de sus hogares.

Existe incidencia significativa entre los factores culturales y violencia contra la mujer en casos atendidos del Centro Emergencia Mujer Huancané 2019 porque, como resultado principal, se obtuvo que los estereotipos de género, con un 27,0\% inciden en la violencia contra la mujer, según la prueba estadística de Chi cuadrado, con probabilidad de error del 5\% , con un valor de xc $2(12,000) \geq(1.0636)=4 \mathrm{gl}$, bajo un nivel de significancia de $(\mathrm{p}<0.05)$, como se evidencia en la tabla $\mathrm{N}^{\circ} 05$, donde el $27,0 \%$ indica que los estereotipos conllevan a que se genere desigualdad, se ubique a la mujer en menor nivel y justifique la violencia contra la mujer dentro de sus hogares.

\section{Referencias bibliográficas}

Arenas-rojas, A.R (2014).”Detección de sexismo ambivalente en estudiantes de bachillerato mexicanos”. México

Cárdenas, m., G.A. (2010). "Inventario de sexismo ambivalente: adaptación, validación y relación con variables psicosociales. Salud y sociedad".

Centro de la mujer peruana, Flora Tristán. (2015) La violencia contra la mujer: feminicidio en el perú”. Lima. 
Condori, M. (2010). "Factores individuales, sociales y culturales que influyen en la violencia de la mujer de 20 a 64 años de edad en el centro de salud Ganimedez". Lima.

Condori, M. C. (2015). "Factores culturales y violencia contra la mujer en familias usuarias del cem carabaya. Puno: Universidad Nacional Del Altiplano”. Puno

De la cruz, P. Y., \& M (2015). “Comparación de las actitudes hacia el machismo entre las mujeres del asentamiento humano el vallesito y la comunidad Vvilla Rica” de Lima este”. Lima

Donado, M. (2010). "Sexismo, rasgos de personalidad, y síntomas psicopatológicos en adolescentes colombianos (tesis de doctorado)”. España

Dongo M., a. (2009). "Significado de los factores sociales y culturales en el desarrollo cognitivo”. Brasil

Euston. (2012). Rol de género.

Garcia, C, \& S. (2013).” Estereotipo dela paternidad e identidad de género en adolescentes de la ciudad de mexico. Iiberoamericana de psicologia: ciencia y tecnologia”. México

García, C \& H (2014). "Campos discursivos sexistas: estado del conocimiento”.México

Glick, P \& F (2009).”An ambivalent alliance: hostile and benevolent sexism as complementary justifications for gender inequality”.

Hernandez F. y B. (2015). "Metodologia de investigacion”.Mexico.

Hernandez y B. (2014). "Metodologia de investigacion”. Mexico

Hernández, r (2014). "Metodología de la investigación. 6ta edición. Mc graw hill education”. México.

Herrera P y A. (2010). "Consumo de alcohol y violencia doméstica contra las mujeres: un estudio con estudiantes universitarias de México”. México

Informe $n^{\circ}$ 04-2010/dp-adm. (2010). "Io en el perú: estudio de expedientes judiciales. Defensoria del pueblo".Perú

Instituto nacional de estadística e informática (2017): "brechas de género 2017”Lima.

Jimenez,V., \& T (2016). "Factores culturales de la pareja y la violencia a la mujer del programa "vaso de leche" del distrito de puyca - la unión - 2016». Arequipa.

Lagarde, M. (2009). "Identidad femenina". Mexico.
Larrain H, s. (2013). "Violencia puertas abiertas”. Universitaria s.a.

Larrain, S. (2010)"Violencia en la familia y transmisión”.

Larraín, S. (2014). "Violencia puertas adentro: la mujer golpeada”. Chile: universitaria

Laureano, a. (2009)." Analisis sobre la manifestacion del fenomeno sexismo en la excuela”.

Ley $\mathrm{N}^{\circ}$ 30364, (2015) "Ley para prevenir, sancionar y erradicar la violencia contra las mujeres y los integrantes del grupo familiar”. Lima

Lujan, M. (2013).” Violencia contra las mujeres y alguien más...”. Valencia

Mamani, R. (2018). "Puno, la tercera región del país con más casos de violencia contra la mujer. Diario la república, p 4". Lima

MIMP. (2014). Folleto sobre conceptos fundamentales para la transversalización del enfoque. Perú

MIMP-PNVFS. (2015). "Manual de intervención. Lima, perú: ministerio de la mujer y poblaciones vulnerables y comisión nacional para el desarrollo y vida”.Lima

Ministerio de la Mujer y Poblaciones Vulnerables MIMP (2015) Conceptos fundamentales para la transversalización del enfoque de género. Lima Perú

Ministerio de la mujer y poblaciones vulnerables (2016). "Perú. Violencia basada en género". Lima

Ministerio de la mujer y poblaciones vulnerables. (2012). "Conceptos fundamentales sobre el enfoque de género para abordar políticas públicas”. Perú

Molina, E (2019). "Factores de riesgo y consecuencias de la violencia de género en Colombia”. Bogotá Colombia

Moral, J. \& R. (2016) "machismo, victimización y perpetración en mujeres y hombres mexicanos. Estudios sobre la cultura contemporánea”.

Navas, M \& G(2016). "Salud, familia y binestar. España”.España.

Ñaupas et. Al, .. (2014). "Metodologia de la investigacion cuantitativa - cualitativa y redacción de tesis “.

Organización mundial de la salud. (2012). "Violencia contra la mujer".

OMS (2005). "Estudio multipaís de la oms sobre salud de la mujer y violencia doméstica 
contra la mujer".Ginebra.

OMS (2010). "Prevención de la violencia sexual y violencia infligida por la pareja contra las mujeres”. Perú

Organización de las Naciones Unidas (2016): "Informe mundial sobre la violencia en el mundo".

Organización Mundial De Salud (2013):”Informe sobre violencia contra la mujer". Lima

Peru 21. (2016). "Informe de la mesa de género de la cooperación internacional. Noticia” Puno.

Peru21. (2016). "Esta es la brecha salarial entre hombres y mujeres en el Perù". Puno

Rodríguez, A y O. (2017). "Métodos científicos de indagación y de construcción del conocimiento". Cuba.

PNUD. (2012). "Estrategia de igualdad de género pdnu”. Perú

Quesada, J., \& L. (2010). "Estereotipos de género y usos de la lengua: un estudio en educación secundaria”. Albacete.

Ragneda, M. (2012). "Trayectorias medios de comunicación masiva y la mujer en italia: de la violencia simbólica a la violencia física”.

RAE. (2001). "Diccionario de la lengua española”. Madrid

Ramos, M. (2013). "Violencia familiar. Protección de la víctima frente a las agresiones”. Perú.

Ruiz. P (2014) "Una aproximación al concepto de género. En defensoría del pueblo sobre género, derecho y discriminación”. Lima

Suárez, J. (2011). "Estereotipos de la mujer en la comunicación".

Vargas, R. (2017).”Factores socioculturales que influyen en la violencia intrafamiliar a partir de la diferencia de género en el distrito Huancayo en la actualidad". Huancayo.

Velázquez, W. (2010).” Género roles de género en la sociedad. Gobierno de Chile". Chile.

Villanueva,V \& M. (2015). "Factores socio culturales y económicos que condicionan la violencia contra la mujer en el centro de salud del distrito caracoto - puno 2015”. Puno. 